\title{
Whole Blood Coagulation and Platelet Activation in the Athlete: A Comparison of Marathon, Triathlon and Long Distance Cycling
}

\author{
A. A. Hanke ${ }^{1}$, A. Staib ${ }^{1}$, K. Görlinger ${ }^{2}$, M. Perrey ${ }^{1}$, D. Dirkmann², P. Kienbaum ${ }^{1}$ \\ ${ }^{1}$ Klinik für Anästhesiologie, Universitätsklinikum Düsseldorf, Germany, \\ ${ }^{2}$ Klinik für Anästhesiologie und Intensivmedizin, Universitätsklinikum Essen, Germany
}

\begin{abstract}
Introduction: Serious thrombembolic events occur in otherwise healthy marathon athletes during competition. We tested the hypothesis that during heavy endurance sports coagulation and platelets are activated depending on the type of endurance sport with respect to its running fraction.

Materials and Methods: 68 healthy athletes participating in marathon (MAR, running $42 \mathrm{~km}, \mathrm{n}=24$ ), triathlon (TRI, swimming $2.5 \mathrm{~km}+$ cycling $90 \mathrm{~km}+$ running $21 \mathrm{~km}, \mathrm{n}=22$ ), and long distance cycling (CYC, $151 \mathrm{~km}, \mathrm{n}=22$ ) were included in the study. Blood samples were taken before and immediately after completion of competition to perform rotational thrombelastometry. We assessed coagulation time (CT), maximum clot firmness (MCF) after intrinsically activation and fibrin polymerization (FIBTEM). Furthermore, platelet aggregation was tested after activation with ADP and thrombin activating peptide 6 (TRAP) by using multiple platelet function analyzer.

Results: Complete data sets were obtained in 58 athletes (MAR: $\mathrm{n}=20$, TRI: $\mathrm{n}=19$, CYC: $\mathrm{n}=19$ ). CT significantly decreased in all groups (MAR $-9.9 \%$, TRI $-8.3 \%$, CYC $-7.4 \%$ ) without differences between groups. In parallel, MCF (MAR $+7.4 \%$, TRI $+6.1 \%$, CYC $+8.3 \%$ ) and fibrin polymerization (MAR $+14.7 \%$, TRI $+6.1 \%$, CYC $+8.3 \%$ ) were significantly increased in all groups. However, platelets were only activated during MAR and TRI as indicated by increased AUC during TRAP-activation (MAR +15.8\%) and increased AUC during ADP-activation in MAR $(+50.3 \%)$ and TRI $(+57.5 \%)$.

Discussion: While coagulation is activated during physical activity irrespective of type we observed significant platelet activation only during marathon and to a lesser extent during triathlon. We speculate that prolonged running may increase platelet activity, possibly, due to mechanical alteration. Thus, particularly prolonged running may increase the risk of thrombembolic incidents in running athletes.
\end{abstract}

\section{INTRODUCTION}

Popularity of marathon running has increased during the last decade. Today, out of 17 million non professional runners in Germany, 100.000 runners partici- pate in marathon competitions. Thrombembolic events have been reported in marathon athletes during competition accounting for considerable morbidity and mortality in otherwise healthy subjects even in the most experienced runners [1-7]. In previous studies, activation of coagulation and platelets were demonstrated during marathon [8-23]. Although during other endurance sports heavy exercise is performed for several hours severe thrombembolic events occur only very rarely. Accordingly, we tested the hypothesis that coagulation and platelet activation are influenced by the type of endurance sport with respect to different running fractions.

\section{Material And Methods}

The study protocol was approved by the University Hospital Düsseldorf ethic committee and is consistent with the Revised Helsinki Declaration and European Unions Convention on Human Rights and Biomedicine. The study was conducted at the Toyota Yvel Düsseldorf Marathon 2007 (42 km running), Cologne Classic Triathlon 2007 (2.5 km swimming, $90 \mathrm{~km}$ cycling, $21 \mathrm{~km}$ running) and the Remscheid Round 2007 (151 km cycling).

\section{PARTICIPANTS}

68 male volunteers (marathon $(\mathrm{MAR}) \mathrm{n}=24$; triathlon $(T R I) n=22$; cycling $(C Y C) n=22$ ) participated in the study after oral and written information and written consent.

All volunteers were requested to complete a questionnaire providing information on medical and training status. Athletes with manifestation of coagulopathies, previous thromboembolic incidents, or medication altering coagulation or platelet function were excluded from the study.

\section{BLOOD SAMPLES}

Blood samples were taken from a forearm vein using a $0.9 \mathrm{~mm}$ diameter needle and were collected in tubes containing either citatrate or hirudin for anticoagulation (Vacutainer, Becton Dicinson, Heidelberg, Germany) immediately before and after completion of 
competition. Citrated tubes were assigned with rotational thrombelastometry, hirudin tubes were assigned with aggregometry.

Platelet count, hemoglobin concentration, hematocrit, and leukocyte count were measured by an automated hematology analyzer (Sysmex mo. KX-21N, Norderstedt, Germany).

\section{WhOLE BLOOD COAgUlation}

Whole blood coagulation was assessed by rotational thrombelastometry (ROTEM, Pentapharm, Munich, Germany) which is a modification of the thrombelastograph system [24]. ROTEM analyses correlate well with conventional thrombelastography. By using a ball-bearing stabilization ROTEM is less sensitive against mechanical stress and agitation than classical thrombelastography. Accordingly, ROTEM shows good reproducibility and precision during bed site application. Analysis can be performed on four channels at the same time testing different activators of coagulation. Whole blood coagulation was determined after intrinsically activation by calcium chloride, phospholipids and ellagic acid (INTEM assay). Fibrin polymerization was assessed after activation by calcium chloride and tissue factor with addition of Cytochalasin D to disable platelet function and display fibrin polymerization only (FIBTEM assay - both assays Pentapharm, Munich, Germany). We measured coagulation time (CT, times from beginning of the reaction until start of clot formation corresponding to reaction time in classical thrombelastography) and maximum clot firmness (MCF, clot stability at its highest degree which is equivalent to maximum amplitude).

\section{Platelet Function}

Platelet function was assessed using a multiple platelet function analyzer (Multiplate, Dynabyte, Munich, Germany). While platelets are non-thrombogenic in the resting state, they expose receptors when activated by specific agents. Multiplate test cells are constructed with two pairs of sensor wires serving as internal control. After platelet activation ex vivo platelets coat these sensor wires of the analyser. The extent of coating is determined by increased electrical resistance between these wires. Alterations in resistance are recorded continuously as a measure of platelet activation. Accordingly, the degree of platelet aggregation is quantified by planimetry of the area under this curve (AUC) indicating overall platelet activity. For assessment of platelet function platelets were activated by thrombin activating peptide (TRAP-6, TRAPtest) and by adenosine-di-phosphate (ADP, ADPtest, both assays Dynabyte, Munich, Germany).

\section{STATISTICAL ANALYSIS}

Data are expressed as means $( \pm$ standard deviation, SD). The Statistical Package for Social Sciences (SPSS for Windows, 13.0, SPSS Inc., Chicago, IL., USA) and GraphPad Prism (Version 4.02, GraphPad Software Inc., San Diego, CA., USA) were used. Data were positively tested for normal distribution and were then analyzed using paired student's t-Tests for comparison before and after competition and analysis of variance with Bonferroni correction for comparing the impact of the type of sport. A p-value below 0.05 was considered to be significant.

\section{RESULTS}

Complete data sets were obtained in 58 athletes (MAR: $\mathrm{n}=21$, TRI: $\mathrm{n}=19$, CYC: $\mathrm{n}=19$ ). Exclusion from the study was caused by early termination of competition or a time delay with excessive fluid resuscitation before blood sampling after completion of competition.

Demographic data including age and weekly hours of physical activity as well as hemogram results are displayed in Table 1. Significant differences between groups were found for age, but not for weekly training hours. No significant differences were found between haemoglobin levels pre und and post competition in all groups. Platelet concentrations were significantly altered during MAR and TRI without reaching statistical significance between groups. A significant increase of leukocyte levels was found in all groups with a significant higher increase in MAR and TRI.

Table 1. Demographic data of study participants.

\begin{tabular}{|c|c|c|c|c|}
\hline & Marathon & Triathlon & Cycling & $p(A N O V A)$ \\
\hline Age (years) & $43 \pm 8.4$ & $36.5 \pm 6.3$ & $41.7 \pm 12.2$ & $0.0429 *$ \\
\hline Physical activity (hours/week) & $7.9 \pm 9.3$ & $11.1 \pm 5.0$ & $12.2 \pm 9.8$ & 0.2081 \\
\hline Hemoglobin before $(\mathrm{g} / \mathrm{l})$ & $15.0 \pm 0.8$ & $15.7 \pm 0.9$ & $15.1 \pm 1.2$ & 0.1281 \\
\hline Hemoglobin after $(\mathrm{g} / \mathrm{l})$ & $15.2 \pm 0.7$ & $15.5 \pm 0.9$ & $15.5 \pm 1.6$ & 0.9234 \\
\hline Platelets before $\left(\mu \mathrm{l}^{-1}\right)$ & $239.3 \pm 36.5$ & $224.5 \pm 43.2$ & $250.1 \pm 62.4$ & 0.6103 \\
\hline Platelets after $\left(\mu \mathrm{l}^{-1} \mathrm{l}\right)$ & $286.0 \pm 42.5^{\#}$ & $261.3 \pm 51.6 \#$ & $247.9 \pm 62.4$ & 0.0650 \\
\hline Leucocytes before competition $\left(\mu \mathrm{l}^{-1}\right)$ & $5.3 \pm 1.0$ & $5.9 \pm 1.1$ & $5.5 \pm 1.2$ & 0.2331 \\
\hline Leucocytes after competition $\left(\mu \mathrm{l}^{-1} \mathrm{l}\right)$ & $16.8 \pm 3.2^{\#}$ & $16.6 \pm 3.8^{\#}$ & $11.4 \pm 3.2^{\#}$ & $<0.0001 *$ \\
\hline
\end{tabular}

Data are displayed as mean \pm standard deviation. $\mathrm{p}<0.05$. 
Table 2. Results of Thrombelastometry (ROTEM $\left.{ }^{\circledR}\right)$ and impedance aggregometry (Multiplate $\left.{ }^{\circledR}\right)$ before and after competition.

\begin{tabular}{|c|c|c|c|c|}
\hline & Marathon & Triathlon & Cycling & $p(A N O V A)$ \\
\hline \multicolumn{5}{|c|}{ ROTEM ${ }^{\circledR}$ Whole blood coagulation results } \\
\hline CT INTEM before (s) & $172 \pm 15.3$ & $168.1 \pm 12.9$ & $164.7 \pm 17.7$ & 0.3956 \\
\hline CT INTEM after (s) & $155 \pm 18.3 \#$ & $154.2 \pm 11.3^{\#}$ & $152.5 \pm 13.0 \#$ & 0.9255 \\
\hline MCF INTEM before (mm) & $58.1 \pm 3.9$ & $56.1 \pm 3.2$ & $59.3 \pm 5.0$ & 0.0823 \\
\hline MCF INTEM after (mm) & $62.4 \pm 3.8^{\#}$ & $59.5 \pm 3.1^{\#}$ & $64.2 \pm 4.2 \#$ & $0.0013 *$ \\
\hline MCF FIBTEM before (mm) & $10.9 \pm 2.7$ & $8.9 \pm 2.8$ & $9.6 \pm 3$ & 0.0928 \\
\hline MCF FIBTEM after (mm) & $12.5 \pm 3 \#$ & $9.9 \pm 2.4 \#$ & $11.3 \pm 2.4^{\#}$ & $0.0136 *$ \\
\hline \multicolumn{5}{|c|}{ Multiplate ${ }^{\circledR}$ impedance aggregometry results } \\
\hline AUC TRAP activation before $(\mathrm{g} / \mathrm{l})$ & $899 \pm 149$ & $911 \pm 233$ & $970 \pm 186$ & 0.4672 \\
\hline AUC TRAP activation after $(\mathrm{g} / \mathrm{l})$ & $1041 \pm 209 \#$ & $1009 \pm 315$ & $906 \pm 288$ & 0.3492 \\
\hline AUC ADP activation before (g/l) & $529 \pm 174$ & $508 \pm 178$ & $589 \pm 160$ & 0.1281 \\
\hline AUC ADP activation after $(\mathrm{g} / \mathrm{l})$ & $795 \pm 258 \#$ & $800 \pm 320 \#$ & $627 \pm 219$ & 0.0865 \\
\hline
\end{tabular}

Data are displayed as mean \pm standard deviation. $(\mathrm{p}<0.05)$ Note, that results, obtained by thrombelastometry, show a significant increase of clot stability and shortening of coagulation times, while platelet aggregation is increased only in MAR and TRI after ADP activation, respectively in MAR after TRAP activation, but not in CYC. (* mark significant differences between groups, \# mark significant differences as compared to baseline values)
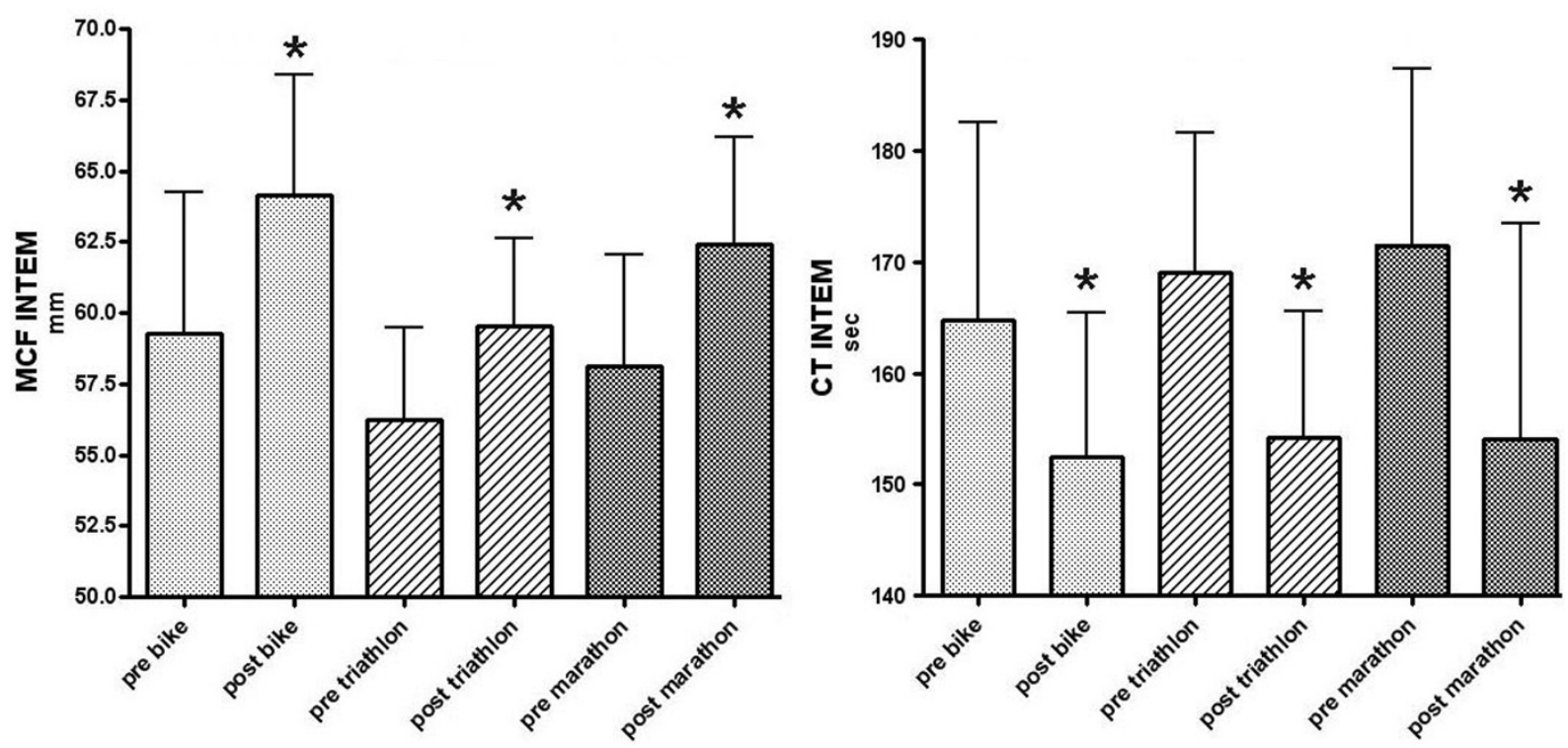

Fig. 1. Results of rotational thrombelastometry after intrinsically activation before and after competition. Maximum clot firmness (MCF) significantly increased in all groups (left panel). Clotting times (CT) significantly decreased in all groups (right panel). ${ }^{*} \mathrm{p}<0.05$.

After intrinsically activation clotting times (CT) significantly decreased in whole blood coagulation in MAR $(-9.9 \%)$, TRI $(-8.3 \%)$, and CYC (-7.4\%) during competition. In parallel, MCF after intrinsically activation increased in all groups (Fig. 1) with a greater increase in MAR $(+7.4 \%)$ and CYC $(+8.3 \%)$ than in TRI $(+6.1 \%)$ (Table 2$)$. Fibrin polymerization significantly increased in all groups (Fig. 2) with a significant larger increase in MAR $(+14.7 \%)$ and CYC $(+17.7 \%)$ than in TRI $(+11.2 \%)$.
Platelets aggregation ex vivo was enhanced depending on the duration of running. After completion of competition platelet aggregation was significantly increased in MAR after both TRAP (+15.8\%) and $\mathrm{ADP}(+50.3 \%)$ activation. In TRI aggreagation increased after ADP activation only $(+57.5 \%)$. In CYC no activity dependent differences in platelet aggregation were recognized (TRAP -6.6\%, ADP $+6.5 \%$ ) (Table 2/Fig. 3). 


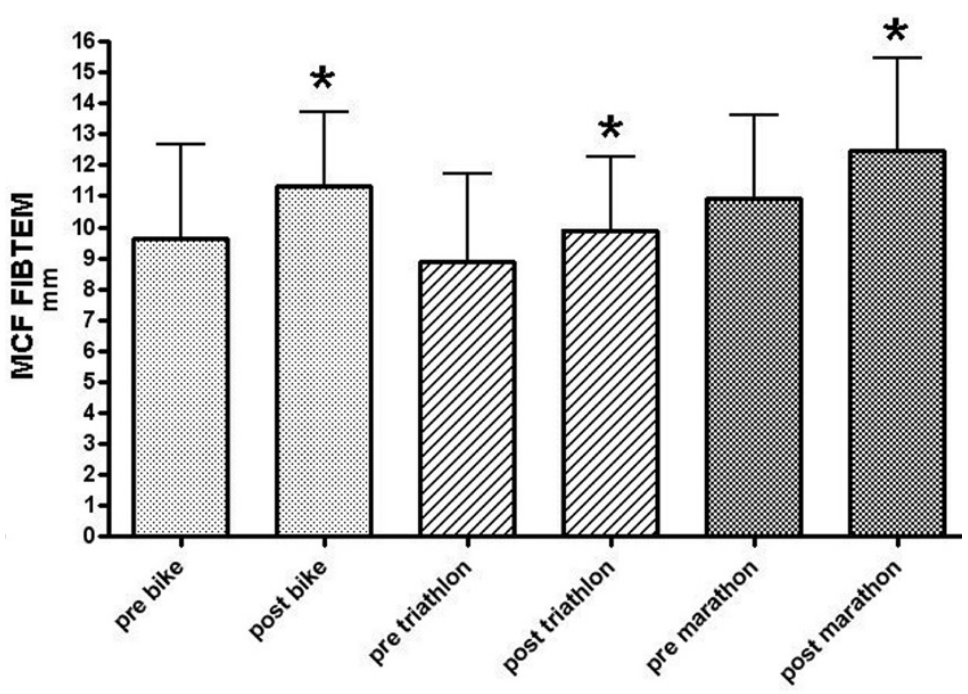

Fig. 2. Maximum clot firmness in fibrin polymerization before and after competition as assessed by rotational thrombelastometry (FIBTEM assay). Results significantly increased in all groups. ${ }^{*} \mathrm{p}<0.05$.
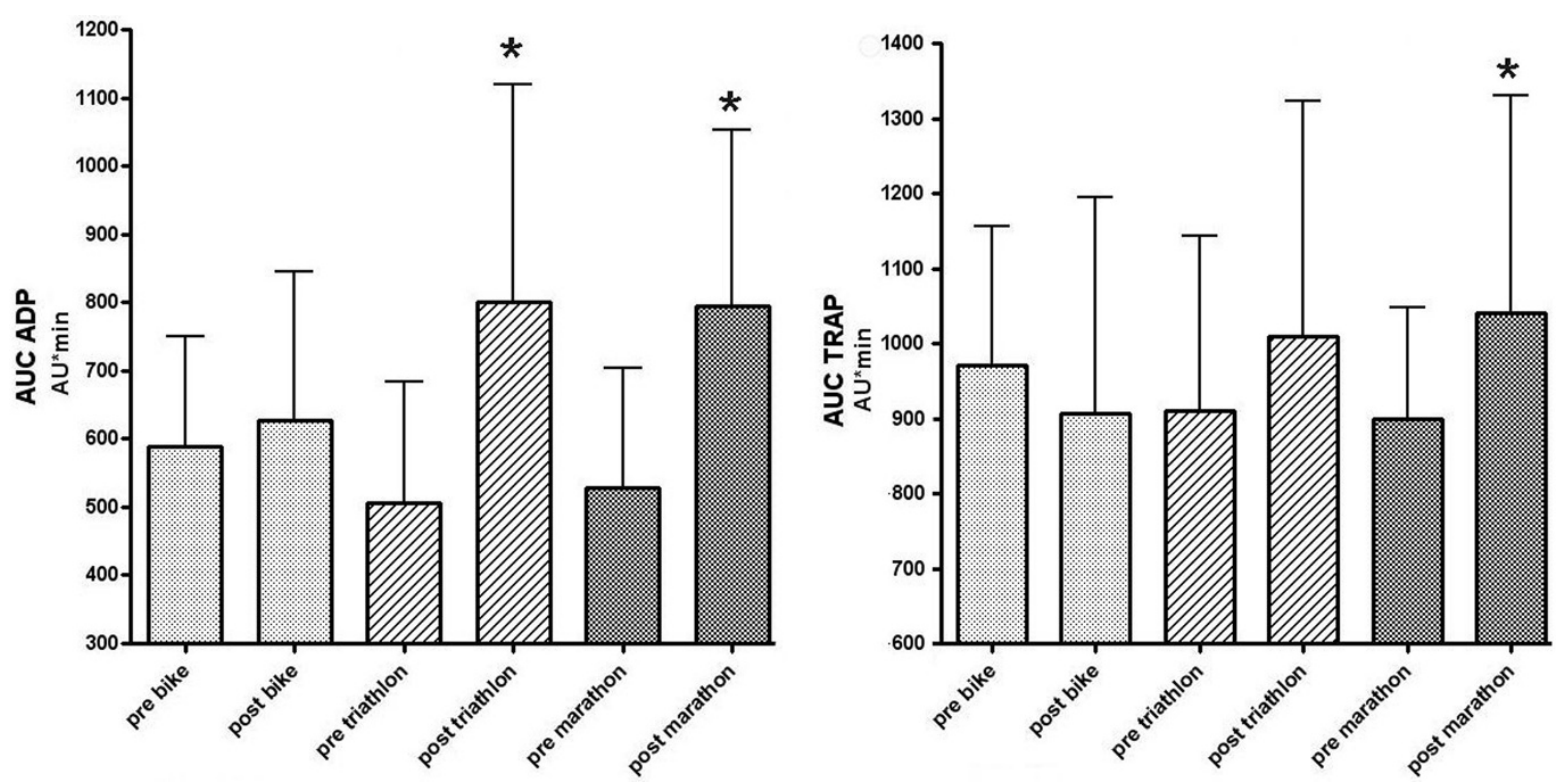

Fig. 3. Results of platelet aggregation after stimulation by adenosine-di-phosphat (ADP, left panel) and thrombin activating peptide 6 (TRAP, right panel) as assessed by multiple platelet function analysis before and after competition. Note, that significant increases between pre and post competition $(* \mathrm{p}<0.05 \mathrm{t})$ are found in marathon after ADP and TRAP activation and in triathlon after ADP activation only, indicating that platelet activation during prolonged activity is dependent on its running fraction.

\section{DisCUSSION}

After completing the first "marathon-run" ever from Marathon to Athens 2500 years ago, Pheidippides died $[25,26]$. Today morbidity and mortality during marathon running are still considerably. Mortality varies with age from $0.3 / 100.000$ in young athletes $[27,28]$ to $7 / 100.000$ in elderly runners [29]. An analysis of the London marathon (650.000 completed runs) indicated an average cardiac death rate of 1.25/100.000 [30]. Although the most frequent disease associated with deaths during marathon is previously unrecognized coronary artery disease $[30,31]$ other thrombembolic incidents have been reported as well 1-7. Our data further emphasize the clinical significance of previously demonstrated activation of the coagulation system during excessive physical exercise [7-13, 15-19, 21-23, 32].

The results of our study demonstrate that whole blood coagulation is markedly altered during heavy en- durance sports irrespective of the running fraction. In contrast, elevated platelet aggregation is observed particularly during marathon and to a lesser extent also during triathlon. We speculate that mechanical stress during running may trigger platelet activation. Thus, the higher incidents of severe thrombembolic incidents in otherwise healthy athletes during marathon compared to triathlon or long distance cycling may be explained by a marked activation of coagulation and platelets at the same time. Influences like venous insufficiency [33] may also contribute to a greater risk in runners.

Changes in hemostatic and fibrinolytic markers during marathon running were discovered more than 30 years ago [12]. At that time the authors demonstrated a significant shortening of partial thromboplastin time while plasma fibrinogen levels and prothrombin times were not altered. By finding shortened euglobulin lysis times and increased levels of fibrin degradation prod- 
ucts the conclusion was made that during marathon coagulation and fibrinolysis are globally activated. In addition, the concentration of prothrombin fragment $1+2$ concentration as well as thrombin-antithrombin complexes in plasma increased indicating activated coagulation. Finally, the plasma concentrations of tissue plasminogen activator antigen, plasminogen activator inhibitor type 1 antigen, d-dimer and plasma fibrinogen degradation products increased in parallel confirming activated fibrinolysis [15]. Accordingly, both coagulation and fibrinolytic activation occurs during a marathon run at the same time.

In contrast, a two hour treadmill exercise at an individual anaerobic threshold of 90\% [10] showed only a small increase in thrombin generation markers, but a distinct increase in fibrinolysis. While moderate exercise led to an increase in plasmin formation only, very heavy exercise also inducted thrombin and fibrin generation balanced by an increase in plasmin generation [22]. Similar results were reported for two hour triathlon demonstrating a moderate activation of coagulation as indicated by thrombin and fibrin formation accompanied by markedly increased plasmin formation [34]. Thus, during exercise free of heavy running periods activation of coagulation and fibrinolysis does not occur in favour of increased fibrinolysis.

The effect of endurance sports on platelets and their activation was assessed during marathon by Rock [16] and Dimitradou [9], during triathlon by Mockel [14] and during a cycle ergometer test by Li [35]. All studies report on platelet activation during physical activity, but a comparison of these sports using identical methods had not been performed previously. While all these studies were able to demonstrate platelet activation during heavy exercise they were not designed to distinguish between various disciplines and did not relate these findings to variables of coagulation.

While most studies on coagulation changes during physical activity assessed more or less isolated variables describing coagulation by measuring single clotting factors or decomposition products, rotational thrombelastometry analyzes both, clotting times and the stability of the clot over the time. Since rotational thrombelastometry is performed in whole blood, its results are affected by interaction of platelets, clotting factors, fibrin polymerization and fibrinolysis, giving information about the end product of coagulation, the clot itself, displaying overall coagulation activity. This method was validated in a model of systemic activation of fibrinolysis and coagulation [20]. Whole blood coagulation in marathon runners demonstrated decreased clotting times and increased clot stability in runners after participating a downhill marathon [21]. Our results are consistent with these alterations in coagulation after intrinsically activation. However, fibrin polymerization was not tested and whole blood coagulation in other endurance sports has not been analyzed to date. The INTEM assay was chosen with regard to the previously published study to confirm their findings during a marathon and to compare these findings to other endurance sports. The FIBTEM assay allows excluding platelet function from the whole blood coagulation assay and displays fibrin polymerization only. Since fibrinogen is an acute phase protein and inflam- mation during heavy exercise is a matter of discussion we decided to include this particular test into our study. Platelet function itself is not accurately mirrored during ROTEM analysis. Accordingly, we evaluated platelet function by multiple platelet function analysis (Multiplate, Dynabyte, Munich, Germany). For reproduction of results from platelet activation during a marathon after ADP activation which were previously described [9, 16, 21, 35] we activated platelets with ADP. Since TRAP-6 is recognized as an even stronger platelet activator we also decided to include TRAP-6 activation.

Clotting times in rotational thrombelastometry mirror activity of coagulation factors and were shortened throughout the tested groups indicating an increase of coagulation factor activity during heavy physical exercise. In contrast, maximum clot firmness is mostly influenced by fibrin polymerization and platelet function. In a perioperative setting it is shown that elevated results of maximum clot firmness in thrombelastometry strongly correlate with the incidence of postoperative thromboembolic events and myocardial infarction [36]. Furthermore, increased platelet activation also elevates the thrombembolic risk [37, 38]. We found whole blood coagulation parameters increased in all tested groups, while platelet aggregation was altered during marathon and to a lesser extent during triathlon but not during cycling, conclude that the running fraction is responsible for platelet activation, while an activation of the plasmatic coagulation system is triggered by physical activity itself. Strenuous exercise activates coagulation probably due to inflammatory effects as shown by previously published studies [21, 39]. We found increasing leukocyte levels in all groups matching these results. Greater increases of leukocyte levels in marathon and triathlon might indicate greater inflammatory effects of these endurance sports. Moreover, we speculate that comparable to effects of mechanical stress during prolonged running on red blood cells leading to hemolysis [40-42], direct mechanical stress on circulating platelets during running contributes to the observed platelet activation.

\section{LIMITATIONS}

Participants in the cycling group were slightly older compared to the marathon and triathlon group. However, there are no indicators that coagulation and fibrinolysis may be different at baseline when comparing subjects at age 41 and age 35 . Training status may flaw baseline coagulation and fibrinolysis. However, this influence could be excluded since the duration of weekly training periods did not differ between the groups studied. Since we did not evaluate fibrinolysis further studies may clarify whether the enhanced whole blood coagulation and platelet aggregation during ex vivo test may be balanced by increased fibrinolysis.

The use of antithrombotic strategies has been shown to be effective in other not desease related situations of prothrombotic stages like thrombembolic events during air travel [43]. Whether this also accounts for marathon running can not be assessed by our study and should be investigated in further studies. 


\section{SUMMARY AND CONCLUSION}

In summary, we compared whole blood coagulation and platelet aggregation during different forms of strenuous exercise. Irrespective of the type of exercise (marathon, triathlon, long distance cycling) marked activation of coagulation is observed. In contrast, only during marathon and to a lesser extend during triathlon a significant increase in platelet aggregabilty was found. We speculate that platelet activation is induced by mechanical stress on thrombocytes and/or inflammation. Finally, our data may explain the increased risk for thrombembolic events in otherwise healthy subjects particularly during marathon.

The knowledge of running dependent platelet and coagulation activation may trigger an even more careful evaluation of athletes involved in marathon running. Accordingly, future studies are necessary to define variables or tests that may identify athletes at risk for thrombembolic events prior to competition.

Acknowledgement: The authors would like to thank the participating athletes for $5907.5 \mathrm{~km}$ of strenuous endurance sports (1281 km running, $4579 \mathrm{~km}$ cycling, $47.5 \mathrm{~km}$ swimming) that is even further than a trip from New York City to Paris (5851 $\mathrm{km})$.

Competing Interest: None to declare.

Financial Support: Departmental sources

\section{REFERENCES}

1. Chan KL, Davies RA, Chambers RJ. Coronary thrombosis and subsequent lysis after a marathon. J Am Coll Cardiol. 1984;4:1322-1325.

2. Disdier P, Harle, Swiader L, Gambarelli-Mouillac N, Weiller PJ. [Retinal vein occlusions in a marathon runner]. Presse Med. 1992;21:582.

3. Gaudard A, Varlet-Marie E, Monnier JF et al. Exerciseinduced central retinal vein thrombosis: possible involvement of hemorheological disturbances. A case report. Clin Hemorheol Microcirc. 2002;27:115-122.

4. Scobie BA. Gastrointestinal emergencies with marathontype running: omental infarction with pancreatitis and liver failure with portal vein thrombosis. $\mathrm{N}$ Z Med J. 1998;111:211-212.

5. Thompson GR. Grand rounds--Hammersmith Hospital. Hazards of running a marathon. BMJ. 1997;314:10231025.

6. Whitson BA, Nath DS, Knudtson JR, Shumway SJ. Cardiopulmonary bypass in revascularization and fluid management of exercise-induced acute myocardial infarction. J Card Surg. 2006;21:480-483.

7. Markov LN. [The syndrome of disseminated intravascular coagulation in marathon athletes]. Ter Arkh. 1989;61:9092.

8. Arai M, Yorifuji H, Ikematsu S et al. Influences of strenuous exercise (triathlon) on blood coagulation and fibrinolytic system. Thromb Res. 1990;57:465-471.

9. Dimitriadou C, Dessypris A, Louizou C, Mandalaki T. Marathon run II: Effects on platelet aggregation. Thromb Haemost. 1977;37:451-455.

10. Hilberg T, Glaser D, Reckhart C, Prasa D, Sturzebecher J, Gabriel HH. Blood coagulation and fibrinolysis after long-duration treadmill exercise controlled by individual anaerobic threshold. Eur J Appl Physiol. 2003;90:639642.

11. Hilberg T, Prasa D, Sturzebecher J, Glaser D, Schneider $\mathrm{K}$, Gabriel HH. Blood coagulation and fibrinolysis after extreme short-term exercise. Thromb Res. 2003;109:271277.

12. Mandalaki T, Dessypris A, Louizou C, Bossinakou I, Panayotopoulou C, Antonopoulou A. Marathon run I: effects on blood coagulation and fibrinolysis. Thromb Haemost. 1977;37:444-450.

13. Mandalaki T, Dessypris A, Louizou C, Panayotopoulou C, Dimitriadou C. Marathon Run III: effects on coagulation, fibrinolysis, platelet aggregation and serum cortisol levels. A 3-year study. Thromb Haemost. 1980;43:49-52.

14. Mockel M, Ulrich NV, Heller G, Jr. et al. Platelet activation through triathlon competition in ultra-endurance trained athletes: impact of thrombin and plasmin generation and catecholamine release. Int J Sports Med. 2001;22:337-343.

15. Prisco D, Paniccia R, Bandinelli B et al. Evaluation of clotting and fibrinolytic activation after protracted physical exercise. Thromb Res. 1998;89:73-78.

16. Rock G, Tittley P, Pipe A. Coagulation factor changes following endurance exercise. Clin J Sport Med. 1997;7:94-99.

17. Rocker L, Taenzer M, Drygas WK, Lill H, Heyduck B, Altenkirch HU. Effect of prolonged physical exercise on the fibrinolytic system. Eur J Appl Physiol Occup Physiol. 1990;60:478-481.

18. Siegel AJ, Stec JJ, Lipinska I et al. Effect of marathon running on inflammatory and hemostatic markers. Am J Cardiol. 2001;88:918-20, A9.

19. Smith JE, Garbutt G, Lopes P, Pedoe DT. Effects of prolonged strenuous exercise (marathon running) on biochemical and haematological markers used in the investigation of patients in the emergency department. $\mathrm{Br} \mathrm{J}$ Sports Med. 2004;38:292-294.

20. Spiel AO, Mayr FB, Firbas C, Quehenberger P, Jilma B. Validation of rotation thrombelastography in a model of systemic activation of fibrinolysis and coagulation in humans. J Thromb Haemost. 2006;4:411-416.

21. Sumann G, Fries D, Griesmacher A et al. Blood coagulation activation and fibrinolysis during a downhill marathon run. Blood Coagul Fibrinolysis. 2007;18:435440.

22. Weiss C, Seitel G, Bartsch P. Coagulation and fibrinolysis after moderate and very heavy exercise in healthy male subjects. Med Sci Sports Exerc. 1998;30:246-251.

23. Weiss C, Welsch B, Albert $M$ et al. Coagulation and thrombomodulin in response to exercise of different type and duration. Med Sci Sports Exerc. 1998;30:12051210.

24. HARTERT H. Blutgerinnungsstudien mit der Thrombelastographie, einem neuen Untersuchungsverfahren. Klin Wochenschr. 1948;26:577-583.

25. Grogan R. Run, Pheidippides, Run! The story of the Battle of Marathon. Br J Sports Med. 1981;15:186-189.

26. Kinsey TE. Pheidippides and the Marathon run. Br J Sports Med. 1981;15:285-286.

27. Maron BJ, Poliac LC, Roberts WO. Risk for sudden cardiac death associated with marathon running. J Am Coll Cardiol. 1996;28:428-431.

28. Van Camp SP, Bloor CM, Mueller FO, Cantu RC, Olson HG. Nontraumatic sports death in high school and college athletes. Med Sci Sports Exerc. 1995;27:641-647.

29. Siscovick DS, Weiss NS, Fletcher RH, Lasky T. The incidence of primary cardiac arrest during vigorous exercise. N Engl J Med. 1984;311:874-877.

30. Tunstall Pedoe DS. Marathon cardiac deaths : the london experience. Sports Med. 2007;37:448-450.

31. Willich SN, Lewis M, Lowel H, Arntz HR, Schubert F, Schroder R. Physical exertion as a trigger of acute myocardial infarction. Triggers and Mechanisms of Myocardial Infarction Study Group. N Engl J Med. 1993;329: 1684-1690. 
32. van Loon BJ, Heere LP, Kluft C, Briet E, Dooijewaard $\mathrm{G}$, Meinders AE. Fibrinolytic system during long-distance running in IDDM patients and in healthy subjects. Diabetes Care. 1992;15:991-996.

33. Holzheimer RG, Stautner-Bruckmann C. Calf pain in runners may be caused by venous insufficiency. Eur J Med Res. 2008;13:218-220.

34. Bartsch P, Welsch B, Albert M, Friedmann B, Levi M, Kruithof EK. Balanced activation of coagulation and fibrinolysis after a 2-h triathlon. Med Sci Sports Exerc. 1995;27:1465-1470.

35. Li N, He S, Blomback M, Hjemdahl P. Platelet activity, coagulation, and fibrinolysis during exercise in healthy males: effects of thrombin inhibition by argatroban and enoxaparin. Arterioscler Thromb Vasc Biol. 2007;27:407413.

36. McCrath DJ, Cerboni E, Frumento RJ, Hirsh AL, nettGuerrero E. Thromboelastography maximum amplitude predicts postoperative thrombotic complications including myocardial infarction. Anesth Analg. 2005;100:15761583.

37. Davi G, Patrono C. Platelet activation and atherothrombosis. N Engl J Med. 2007;357:2482-2494.

38. McNicol A, Israels SJ. Beyond hemostasis: the role of platelets in inflammation, malignancy and infection. Cardiovasc Hematol Disord Drug Targets. 2008;8:99-117.

39. Cerneca E, Simeone R, Bruno G, Gombacci A. Coagulation parameters in senior athletes practicing endurance sporting activity. J Sports Med Phys Fitness. 2005;45:576579.

40. Davidson RJ. March or exertional haemoglobinuria. Semin Hematol. 1969;6:150-161.

41. Robinson Y, Cristancho E, Boning D. Intravascular hemolysis and mean red blood cell age in athletes. Med Sci Sports Exerc. 2006;38:480-483.
42. Spicer AJ. Studies on march haemoglobinuria. Br Med J. 1970;1:155-156.

43. Ferrari E, Morgan G. Travel as a risk factor for venous thromboembolic disease. Eur J Med Res. 2004;9:146-149.

Received: July 22, 2009 / Accepted: August 14, 2009

Address for correspondence:

Dr. med. Alexander Hanke

Klinik für Anästhesiologie

Klinikum der Heinrich-Heine-Universität Düsseldorf

Moorenstr. 5

40225 Düsseldorf

Germany

E-mail: Alexander.Hanke@med.uni-duesseldorf.de

Andreas Staib

E-mail: email@andy-staib.com

Dr. Klaus Görlinger

E-mail: anke-klaus.goerlinger@t-online.de

Mareike Perrey

E-mail: mareike.perrey@web.de

Dr. Daniel Dirkmann

E-mail: daniel.dirkmann@versanet.de

Prof. Dr. Peter Kienbaum

E-mail: peter.kienbaum@med.uni-duesseldorf.de 